\title{
Generation of inverted heliospheric magnetic flux by coronal loop opening and slow solar wind release
}

Article

Accepted Version

Owens, M. J., Lockwood, M., Barnard, L. A. and Macneil, A. R. (2018) Generation of inverted heliospheric magnetic flux by coronal loop opening and slow solar wind release. The Astrophysical Journal, 868 (1). L14. ISSN 0004-637X doi: https://doi.org/10.3847/2041-8213/aaee82 Available at https://centaur.reading.ac.uk/80704/

It is advisable to refer to the publisher's version if you intend to cite from the work. See Guidance on citing.

To link to this article DOI: http://dx.doi.org/10.3847/2041-8213/aaee82

Publisher: American Astronomical Society

All outputs in CentAUR are protected by Intellectual Property Rights law, including copyright law. Copyright and IPR is retained by the creators or other copyright holders. Terms and conditions for use of this material are defined in the End User Agreement.

www.reading.ac.uk/centaur 
Central Archive at the University of Reading

Reading's research outputs online 
Draft Version SePtember 27, 2018

Typeset using $\mathrm{LATEX}_{\mathrm{E}}$ default style in AASTeX62

Generation of inverted heliospheric magnetic flux by coronal loop opening and slow solar wind release

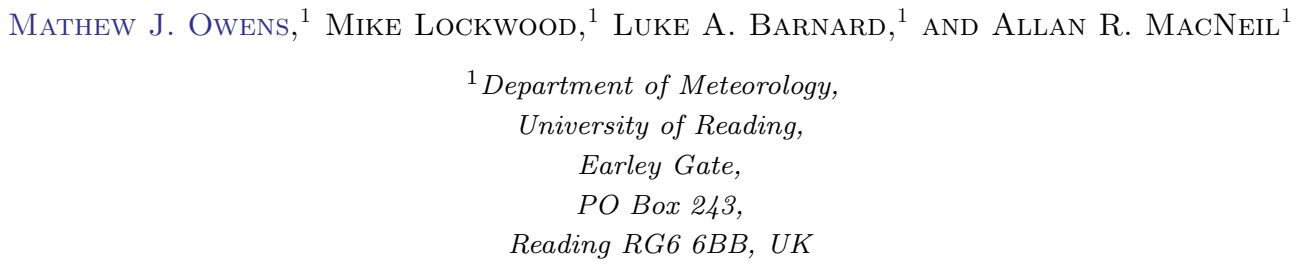

(Received September 27, 2018)

Submitted to ApJL

\begin{abstract}
In situ spacecraft observations can provide much-needed constraints on theories of solar wind formation and release, particularly the highly variable slow solar wind which dominates near-Earth space. Previous studies have shown an association between local inversions in the heliospheric magnetic field (HMF) and solar wind released from the vicinity of magnetically closed coronal structures. We here show that the properties of such inverted HMF observed in situ are consistent with the same coronal source regions as the slow solar wind. We propose that inverted HMF is produced by solar wind speed shear, which results from coronal loop opening via reconnection with an open flux tube and introduces a pattern of fast-slow-fast wind along a given HMF flux tube. This same loop-opening process is thought to be central to slow solar wind formation. The upcoming Parker Solar Probe and Solar Orbiter missions provide a unique opportunity to directly observe these processes and thus determine the origin of the slow solar wind.
\end{abstract}

Keywords: solar wind, heliospheric magnetic field, heliosphere

\title{
1. INTRODUCTION
}

A fraction of the magnetic flux which threads the photosphere reaches sufficient coronal altitude to be dragged out by the solar wind flow to form the heliospheric magnetif flux (HMF, Owens \& Forsyth (2013)). The integrated (unsigned) HMF is also referred to as the open solar flux (OSF). It can be measured in two ways. The observed photospheric magnetic field can be extrapolated up to through the corona using a model, and the OSF estimated as the total unsigned magnetic flux threading a surface at a given altitude (the source surface) (Wang \& Sheeley 1995). Alternatively, the HMF can be directly measured in situ, typically by spacecraft in near-Earth space, and this single-point observation assumed to be representative of the global OSF (Lockwood 2013). While there is qualitative agreement between the two approaches, the in situ estimates are significantly larger than the photospheric magnetic field estimates. The reason for the discrepancy is still not fully understood (Linker et al. 2017), but at least part of the explanation lies in the existence of inverted HMF (Owens et al. 2017). This is magnetic flux which is locally folded, so that a flux tube which has a single intersection with the source surface may thread a heliocentric sphere at, e.g. 1 AU, multiple times. Such inverted HMF can be directly identified by the suprathermal electron strahl, which always moves anti-sunward, in a global sense, along the HMF (Crooker et al. 2004).

In situ HMF observations at increasing radial distance tend to result in increasingly large OSF estimates (Owens et al. 2008), suggesting inverted HMF becomes more prevalent at larger heliocentric distances. This is to be expected, as the increasing angle of the Parker spiral to the radial direction means smaller deviations are required to fold the

Corresponding author: Mathew Owens

m.j.owens@reading.ac.uk 
HMF back towards the Sun. Inverted HMF can be formed by heliospheric processes, such as waves and turbulence, which twist the HMF about the nominal Parker spiral configuration. But inverted HMF has also been shown to preferentially originate from dipolar- and pseudo-streamers; where open and closed coronal flux converge (Owens et al. 2013). This association suggests inverted HMF is also generated as a result of coronal processes.

As fast solar wind originates in relatively cool open flux regions within coronal holes, and slow solar wind is associated with hotter closed coronal loops, inverted HMF may be a result of slow wind formation and release, which is still debated (Crooker et al. 2012; Fisk 2003; Antiochos et al. 2007). In this study, we investigate the elemental composition and ion charge-state properties of inverted HMF, as these provide a diagnostic of coronal source conditions.

\section{DATA AND METHODS}

In this study, we use inverted and uninverted HMF intervals determined by Owens et al. (2017), which combines 64-second ACE magnetic field and suprathermal electron data (McComas et al. 1998; Smith et al. 1998) using the simple algorithm of (Owens et al. 2013). In summary, the mean 272-eV electron flux in the three pitchangle bins centred on 90 pitch angle (i.e., perpendicular to the magnetic field direction) is used to compute the background flux. The background flux is then compared with the mean flux in the three most field-aligned pitch-angle bins (i.e., parallel to the magnetic field) and the mean flux in the three most antiparallel bins. If the parallel and/or antiparallel flux exceeds the background level by $30 \%$, a parallel and/or antiparallel strahl is determined to exist. The radial magnetic field component is then used to determine the strahl direction in the heliospheric frame and hence the HMF topology. If the strahl is antisunward, the HMF is uninverted and, whereas if the strahl is sunward, the HMF must be locally inverted increases the apparent OSF.

This HMF topology information is compared with solar wind composition and ion charge-state information provided by the ACE Solar Wind Ion Composition Spectrometer (SWICS) instrument (Gloeckler et al. 1998), here taken from the 1-h merged dataset at ftp://cdaweb.gsfc.nasa.gov/pub/data/ace/multi. The data cover 1998-01-01 to 2011-06-01. ICMEs are removed from the steady-state solar wind, using the updated Cane \& Richardson (2003) ICME catalogue, available from http://www.srl.caltech.edu/ACE/ASC/DATA/level3/icmetable2.htm. It is necessary to accommodate the different time resolutions of the HMF topology and ion composition/charge-state data. The 1-h HMF topology is determined to be the dominant (i.e. most common) 64-second HMF topology within a given hour, excluding datagaps and undetermined topologies. This gives very good agreement with the stricter requirement of a majority (i.e. at least $50 \%$ ) of the 64-second topologies within a given hour be of a specific type. Over the 1998 to 2011 period with ICMEs removed, this results in 9,359 hourly of inverted HMF and 109,878 hours of uninverted HMF.

We compare the cumulative distribution functions (CDFs) for the inverted and uninverted HMF intervals for each solar wind parameter. To quantify the effect of finite sample size, 9,359 intervals of uninverted HMF are randomly sampled. This is performed 1000 times and compute the 1-, 2- and 3-sigma ranges of the resulting CDFs.

In addition to visual inspection of the CDFs, it is also useful to have a measure of the degree of difference between the inverted and uninverted distributions. We use the Kolmogorov-Smirnov (KS) non-parametric test to quantify the probability that the inverted and uninverted distributions are subsamples of the same underlying distribution. But we also seek a measure of the magnitude of the difference in distributions (i.e., the "effect size"). The strictly standardised mean difference (SSMD) compares the difference in the mean values, $\mu$, of two distributions in terms of their standard deviations, $\sigma$ (thus it is similar to a Fischer Z-score) (Zhang 2010). Assuming distributions are independent, this takes the form:

$$
\beta=\frac{\mu_{1}-\mu_{2}}{\sqrt{\sigma_{1}^{2}+\sigma_{2}^{2}}}
$$

As the distributions of solar wind paramters considered in this study are generally not Gaussian, we instead take a more non-parametric approach, replacing $\mu$ by the median, $m$, and $\sigma$ by half the interquartile range, $I Q R$, giving:

$$
\beta^{\prime}=2 \frac{m_{1}-m_{2}}{\sqrt{I Q R_{1}^{2}+I Q R_{2}^{2}}}
$$

This measure is used in a purely relative sense. However, the thresholds for low, medium and strong effect size of 0 , 0.25 and 1 (Zhang 2010) are useful guides.

\section{RESULTS}

Figure 1 shows the durations of contiguous inverted and uninverted HMF interval. A given interval is defined in hourly data as lasting until a different HMF topology, an uncatagorised hour or a datagap is observed. The inverted 


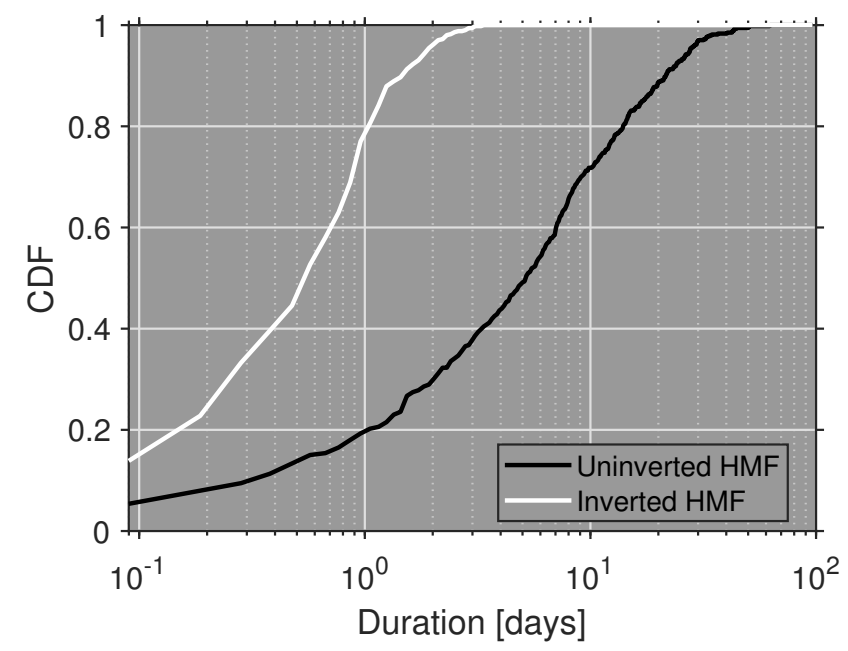

Figure 1. Cumulative distribution functions (CDFs) of the durations of contiguous uninverted (black) and inverted (white) HMF intervals in 1-hour data. Note the log scale on the x-axis.

\begin{tabular}{l|ccc}
$\begin{array}{r}\text { Solar wind } \\
\text { parameter }\end{array}$ & $\begin{array}{c}\beta^{\prime} \text { for all } \\
\text { uninverted HMF }\end{array}$ & $\begin{array}{c}\beta^{\prime} \text { for slow } \\
\text { uninverted HMF }\end{array}$ & $\begin{array}{c}\beta^{\prime} \text { for fast } \\
\text { uninverted HMF }\end{array}$ \\
\hline$|B|$ & -0.52 & -0.44 & -0.68 \\
$\left|B_{R}\right| /|B|$ & -0.50 & -0.42 & -0.60 \\
$|V|$ & -0.42 & 0.1 & $\mathbf{- 2 . 0 0}$ \\
$n_{P}$ & 0.24 & -0.18 & 0.70 \\
$\alpha: \mathrm{p}$ & 0.02 & -0.00 & 0.02 \\
$\mathrm{Fe}: \mathrm{O}$ & -0.02 & -0.26 & 0.28 \\
$\left\langle q_{C}\right\rangle$ & 0.36 & -0.08 & $\mathbf{1 . 1 6}$ \\
$\left\langle q_{O}\right\rangle$ & 0.54 & 0.20 & $\mathbf{1 . 3 0}$ \\
$\left\langle q_{F e}\right\rangle$ & 0.12 & 0.14 & 0.1
\end{tabular}

Table 1. Difference between inverted HMF and different populations of uninverted HMF, for a range of solar wind parameters, as measured by $\beta^{\prime}$. Larger indicate greater difference between the distributions. Absolute values above 1 are shown in bold, below 0.25 are shown in italics.

HMF intervals, have a median duration of around 12 hours and an upper limit of around 3.5 days. The uninverted intervals have a median duration of around 5 days and an upper limit of around 50 days. To maximise the available data and improve statistics, we do not exclude any "buffer" region around the transition from inverted to uninverted HMF (and vice versa).

Figure 2 shows the CDFs for solar wind parameters in 1-hour inverted and uninverted HMF hours. The 1-, 2- and 3 -sigma uncertainty bands resulting from limited sample size have been omitted as they are too small to be visible on the plots. Thus for all solar wind parameters considered, the differences between inverted and uninverted HMF are not simply the result of different sample size. Similarly, for all solar wind parameters, the KS-test rejects the null hypothesis that the inverted and uninverted HMF distributions are subsamples of the same underlying distribution at $\mathrm{p}=0.001$, i.e., the $99.9 \%$ confidence level. However, the magnitude of the differences in the inverted and uninverted distributions varies considerably for different solar wind parameters. Table 1 summarises the $\beta^{\prime}$ values to quantify what can be seen by eye from the CDFs.

As reported by Owens et al. (2017), inverted HMF intervals show significantly weaker $|B|$ than uninverted HMF (panel a). Panel b shows inverted HMF is less radial than uninverted HMF, suggesting that (at 1 AU) inverted HMF is only partially folded back on itself. Panels c and d show that inverted HMF is associated with considerably slower, and to a lesser extent, denser solar wind than uninverted HMF. There is little difference, however, between inverted and uninverted HMF in the available composition measures: While the distributions of $\alpha$ :p and Fe:O are statistically 

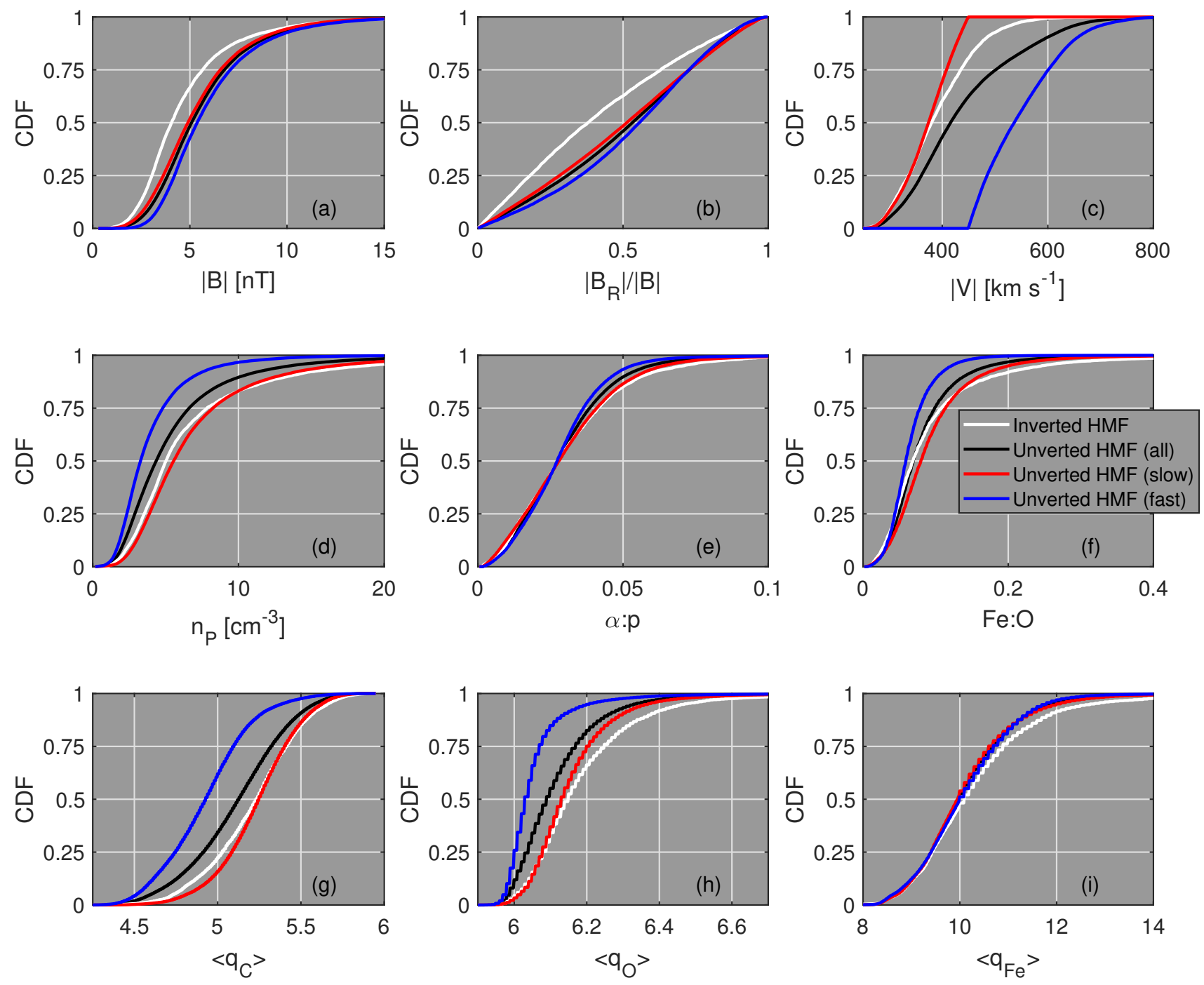

Figure 2. Cumulative distribution functions of solar wind properties of inverted (white) and uninverted (black) HMF intervals. ICMEs have been removed. Fast (blue) and slow (red) intervals of uninverted HMF are also shown.

different, the effect is primarily in the tail of the distributions and the magnitude of the difference is very small, as indicated by the large overlap in the CDFs and the associated small $\beta \prime$ values. Conversely, both the average oxygen and average carbon charge states are significantly elevated in inverted HMF intervals relative to uninverted intervals. For average iron charge states, however, the magnitude of the difference is much smaller and primarily in the tail of the distribution.

Figure 2 and Table 1 also show uninverted HMF further divided into fast and slow wind, respectively, using a threshold of $450 \mathrm{~km} \mathrm{~s}^{-1}$. It is clear that in general, inverted HMF is much more similar to slow wind than fast wind. Indeed, inverted HMF is distinct from fast uninverted HMF in all parameters except $\alpha$ :p. The CDFs of Fe:O for inverted and fast uninverted HMF are clearly separated in the upper part of the distribution, despite the fairly modest value. Inverted HMF is nevertheless distinct from slow wind in terms of the HMF, which is weaker and increasingly inclined the radial direction. 


\section{DISCUSSION AND CONCLUSIONS}

Inverted HMF occurs in short bursts and preferentially in slow, dense solar wind. It generally exhibits lower HMF intensity and increased inclination to the radial direction compared to the solar wind as a whole. We have further shown that inverted HMF is similar to slow solar wind in terms of elemental composition and oxygen, carbon and iron ion charge states, suggesting common coronal source conditions.

Inverted HMF is strongly differentiated from fast uninverted HMF in all parameters except the elemental composition measures and iron ion charge-states. Specifically, inverted HMF is weakly differentiated from fast uninverted HMF in terms of Fe:O abundance ratios but there is little difference in the $\alpha: p$ abundance ratio, as is often the case with the slow wind in general Fu et al. (2017). It is possible that the gradients in abundance are produced in the corona, but that differential streaming of heavy ions relative to the bulk (proton) solar wind (Alterman et al. 2018; Berger et al. 2011) is able to traverse the short inverted HMF intervals and remove this signature by 1 AU (though this should equally wash out the carbon and oxygen charge-state signatures). It may be possible to identify any such compositional signatures using observations from close to the Sun, discussed below, or focusing only the longer-lived HMF inversions, though this prohibits statistical analysis.

Interpretation of ion charge states in terms of coronal temperature is not straight forward, with coronal electron density, temperature and plasma velocities all contributing to the observed values (Zhao et al. 2014). But in general, elevated oxygen and carbon charge states are produced by increased temperatures in the lower corona, whereas elevated iron charge states are more likely to result from heating through the extended corona (Song et al. 2016). Thus inverted HMF is associated with increased heating in the lower corona, which suggests the material was released from hot coronal loops. This is consistent with previous results which show an association between inverted HMF and closed coronal loops in dipolar and unipolar coronal streamers (Owens et al. 2013).

The hot coronal material on closed coronal loops can be released by interchange reconnection (Crooker \& Owens 2011) with an open magnetic flux tube. This could be initiated by either random foot-point motions or more large-scale convection of open flux (Fisk 2003). The inverted HMF observed at 1 AU, however, is unlikely to be formed as a direct result of this reconnection, as shown schematically by Figure 3. We assume that the pre-existing open flux tube is associated with a coronal hole and thus contains low ion charge states and fast solar wind, while the newly opened coronal loop produces enhanced ion charge states and slow solar wind. The inverted magnetic flux that is initially produced by interchange reconnection at point $b$ will be reduced by both the magnetic curvature forces which act to straighten magnetic flux tubes, and by the solar wind speed shear across the inversion. Given the high Alfven speeds in the low corona and the fact that the solar wind speed shear will exist from the moment of reconnection, this magnetic field inversion is unlikely to survive to the upper corona and become a HMF inversion. If the inversion does survive to become part of the solar wind, it would continue to be eroded during transit to $1 \mathrm{AU}$. This runs contrary to the inferred trends in HMF inversions, which suggest a growth with heliocentric distance (Owens et al. 2008), producing an excess flux in the heliosphere (Lockwood et al. 2009) (though this is also the result of the increasing angle of the Parker spiral with heliocentric distance, which means ortho-gardenhose magnetic flux has an increasingly large radial magnetic field contribution).

Instead, we suggest the observed inverted HMF is produced on the non-reconnecting leg of the coronal loop. At some time, $t$, after the reconnection has occurred, this newly opened flux tube will become a source of fast solar wind, particularly if the foot point of the newly open flux tube convects deeper within a pre-existing corona hole (Fisk 2003). This creates a second region of solar wind speed shear over point $a$, this time in a sense to generate inverted magnetic flux, as long as it can overcome the opposing magnetic curvature forces. If the slow/fast winds are not radially aligned, the solar wind speed shear will persist with increasing altitude in the corona, while the Alfven speed will fall off rapidly with height due to declining magnetic field strength. Thus the inverted HMF will grow with increasing radial distance from the Sun, as long as the shear persists. The lifetime of the speed shear will depend on size-scale of slow/fast solar wind bursts associated with this process. Once solar wind speed shear has dissipated, the inverted HMF will be eroded at the local Alfven speed (which itself decreases with heliocentric distance).

The degree of inversion in the HMF at a given radially distance will depend on height profile of the Alfven speed, which can be estimated using a coronal magnetic field model and assumed plasma density profile, the magnitude of the speed sheer and the time for which the sheer has been acting, $t$. By measuring solar wind speed shear, and the strength and occurrence of inverted HMF with radial distance from the Sun, Parker Solar Probe and Solar Orbiter will enable quantification of $t$, which determines how long newly opened coronal loops act as a source of slow solar wind and how quickly they transition to become sources of fast wind. This will provide an observational test of the models 

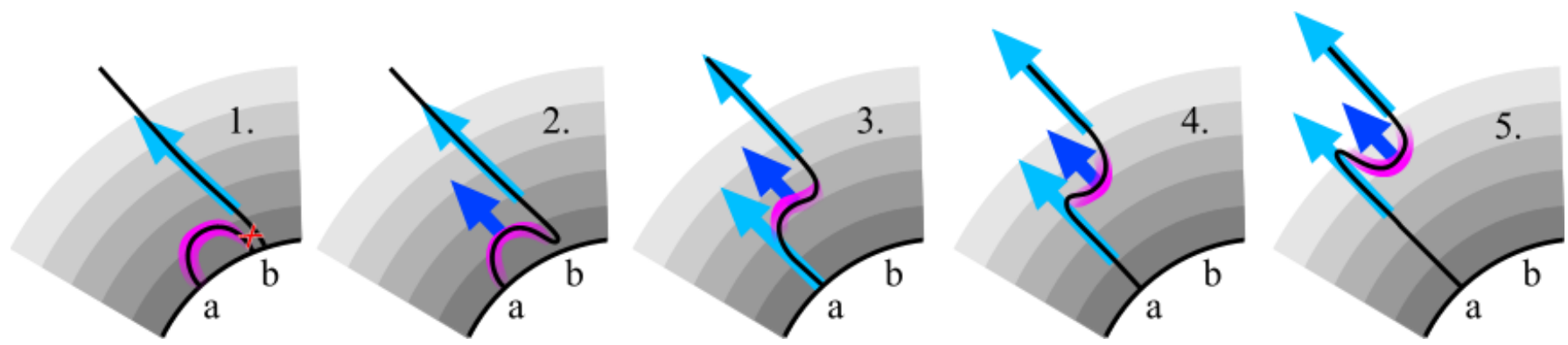

Figure 3. A schematic of inverted HMF formation. Grey shaded bands show increasing altitude through the corona. At time 1 , a closed loop containing hot material (purple shading) reconnects at point $b$ with an open field line containing fast wind (light blue arrow). At time 2, inverted HMF is produced as a result of the reconnection above point b. The hot coronal material begins to rise as newly released slow solar wind (dark blue arrow). At time 3, magnetic curvature forces and solar wind shear combine to remove the HMF inversion above point $b$. The newly open field at point $a$ becomes a source of fast wind. At time 4, the resulting solar wind shear produces new inverted HMF above point $a$. At time 5 , the new inverted HMF grows with increasing altitude.

of slow solar wind generation by continual interchange reconnection at the open/closed magnetic flux boundary (Fisk 2003; Antiochos et al. 2011; Crooker et al. 2012).

\section{ACKNOWLEDGEMENTS}

Work was part-funded by Science and Technology Facilities Council (STFC) grant numbers ST/M000885/1 and ST/R000921/1, and Natural Environment Research Council (NERC) grant number NE/P016928/1. Solar wind composition and ion charge-state information are provided by the ACE Solar Wind Ion Composition Spectrometer (SWICS) instrument, here taken from the 1-hour merged dataset at ftp://cdaweb.gsfc.nasa.gov/pub/data/ace/multi. ACE solar wind magnetic field, plasma and suprathermal electron data were obtained from ftp://cdaweb.gsfc.nasa.gov.

\section{REFERENCES}

Alterman, B. L., Kasper, J. C., Stevens, M. L., \& Koval, A. 2018, The Astrophysical Journal, 864, 112.

http://stacks.iop.org/0004-637X/864/i=2/a=112?key= crossref.70d30c5e4f4e09560b242739d2b64fbe

Antiochos, S. K., DeVore, C. R., Karpen, J. T., \& Miki `'c, Z. 2007, Astrophys. J., 671, 936. http://10.0.4.62/522489

Antiochos, S. K., Miki 'c, Z., Titov, V. S., Lionello, R., \& Linker, J. A. 2011, Astrophys. J., 731, 112. http://10.0.4.64/0004-637X/731/2/112

Berger, L., Wimmer-Schweingruber, R. F., \& Gloeckler, G. 2011, Physical Review Letters, 106, 151103. https: //link.aps.org/doi/10.1103/PhysRevLett.106.151103

Cane, H. V., \& Richardson, I. G. 2003, J. Geophys. Res., 108, 1156. http://10.0.4.5/2002JA009817

Crooker, N. U., Antiochos, S. K., Zhao, X., \& Neugebauer, M. 2012, J. Geophys. Res., 117, 4104. http://10.0.4.5/2011JA017236

Crooker, N. U., Kahler, S. W., Larson, D. E., \& Lin, R. P. 2004, J. Geophys. Res., 109, doi:10.1029/2003JA010278. http://10.0.4.5/2003JA010278

Crooker, N. U., \& Owens, M. J. 2011, Space Science Reviews, 172, 201.

http://link.springer.com/10.1007/s11214-011-9748-1
Fisk, L. A. 2003, J. Geophys. Res., 108, doi:10.1029/2002JA009284. http://10.0.4.5/2002JA009284

Fu, H., Madjarska, M. S., Xia, L., et al. 2017, The Astrophysical Journal, 836, 169. http://stacks.iop.org/0004-637X/836/i=2/a=169?key= crossref.4ffd7820d47fd59f836bcb17bb14c7c3

Gloeckler, G., Cain, J., Ipavich, F. M., et al. 1998, in The Advanced Composition Explorer Mission (Springer), 497-539

Linker, J. A., Caplan, R. M., Downs, C., et al. 2017, Astrophys. J., in press, doi:10.3847/1538-4357/aa8a70

Lockwood, M. 2013, Liv. Rev. Sol. Phys., 10, 4. http://10.0.50.142/lrsp-2013-4

Lockwood, M., Owens, M. J., \& Rouillard, a. P. 2009, Journal of Geophysical Research, 114, 1. http://dx.doi.org/10.1029/2009JA014450

McComas, D. J., Bame, S. J., Barker, S. J., et al. 1998, Space Sci. Rev., 86, 563

Owens, M. J., Arge, C. N., Crooker, N. U., Schwadron, N. a., \& Horbury, T. S. 2008, Journal of Geophysical Research, 113, A12103. http://doi.wiley.com/10.1029/2008JA013677 
Owens, M. J., Crooker, N. U., \& Lockwood, M. 2013, J. Geophys. Res., 118, 1868. http://10.0.3.234/jgra.50259

Owens, M. J., \& Forsyth, R. J. 2013, Liv. Rev. Sol. Phys., 10, 5. http://10.0.50.142/lrsp-2013-5

Owens, M. J., Lockwood, M., Riley, P., \& Linker, J. 2017, J. Geophys. Res., 122, 910. https://agupubs. onlinelibrary.wiley.com/doi/abs/10.1002/2017JA024631

Smith, C. W., L'Heureux, J., Ness, N. F., et al. 1998, Space Sci. Rev., 86, 613
Song, H. Q., Zhong, Z., Chen, Y., et al. 2016, The Astrophysical Journal Supplement Series, 224, 27

Wang, Y.-M., \& Sheeley, N. R. 1995, Astrophys. J. Lett., 447, L143. http://10.0.4.62/309578

Zhang, X. D. 2010, Statistics in Biopharmaceutical Research, 2, 292. http:

//www.tandfonline.com/doi/abs/10.1198/sbr.2009.0074

Zhao, L., Landi, E., Zurbuchen, T. H., Fisk, L. A., \& Lepri, S. T. 2014, The Astrophysical Journal, 793, 44 